\title{
Review
}

Guoxiu Lu*

\section{Research progress on anti-infection therapy for sepsis in children}

DOI: $10.2478 / \mathrm{ii}-2018-0018$

Received April 25, 2018; accepted May 04, 2018; published online June 29, 2018

\begin{abstract}
Sepsis is a systemic inflammatory response caused by infection and a critical illness in pediatrics. This disease is the leading cause of death in infants and children worldwide. An early, appropriate, and adequate anti-infective treatment can effectively prevent disease progression and improve the survival rate of children. However, antimicrobial drug abuse, increased drug-resistant bacteria, and lack of epidemiological data have hampered the effective and rational anti-infective treatment of patients with sepsis and enhancement of the success rate of rescue, especially for children. This article briefly reviews the recent advances in anti-infective treatment for sepsis in children at home and abroad based on sepsis definition, pathogen distribution and drug resistance, infection source control, and rational anti-infection. The results provide a foundation for clinical treatment of sepsis.
\end{abstract}

Keywords: children, sepsis, anti-infection

Sepsis is a common critical illness in pediatrics and the leading cause of death in infants and children worldwide [1]. This disease is characterized by high prevalence rate, high mortality rate, and high treatment costs. The burden of disease by disability-adjusted life years caused by neonatal sepsis and infectious diseases accounted for $1.78 \%$ of the total disability-adjusted life years globally. The incidence of sepsis is predominant in children younger than 5 years, with the highest incidence of 0-6 days after birth, followed by 7-27 days and 28-364 days [2]. Mortality caused by sepsis accounted for $6 \%$ of the total neonatal mortality and $14 \%$ of the total mortality of children [3]. Approximately 260,000 patients have been diagnosed with sepsis in the emergency department in the United States annually. The incidence rate is growing by $0.07 \%$ every 2 years. The mortality rate of sepsis among children is $10.3 \%-12.8 \%$ [4]. The mortality rate of sepsis in China (up to $30 \%$ ) ranks first in the world [5]. Effective and timely control of infection is the key to improving the survival rate of children with sepsis. The international "Save the Sepsis Campaign" has been implemented since 2002. However, the antimicrobial drug abuse and increased drug-resistant bacteria have hampered the ability of many hospitals, especially primary hospitals, to perform prompt etiological inspections or properly select antimicrobial agents based on bacterial culture and drug sensitivity test results. Therefore, the effective and reasonable administration of anti-infective treatment for patients with sepsis and enhancement of the success rate of rescue are facing enormous challenges.

\section{Definition of sepsis}

After the body is infected with pathogens, a series of reactions, such as systemic inflammatory response syndrome, sepsis, severe sepsis, septic shock, acute respiratory distress syndrome, and multiple organ dysfunction syndromes, may occur. In the 2012 edition of the International Guidelines for Treatment of Severe Sepsis and Septic Shock in Children, sepsis is defined as an infection-induced systemic inflammatory response that

Department of Pediatrics, Guangxi Hechi City Hospital, Hechi 546300, China

*Correspondence: Guoxiu Lu, E-mail: LGX6780@163.com 
can progress to severe sepsis (suspected or confirmed focus-induced acute organ dysfunction) and septic shock (severe sepsis accompanied by persistent hypotension and has no effect on fluid resuscitation) [6]. At present, the imbalance of proinflammatory/anti-inflammatory factors is the most important pathogenesis of sepsis. Sepsis treatment includes initial resuscitation, anti-infection, fluid resuscitation, and administration of vasoactive drugs and hormones [7].

\section{Distribution of pathogens}

Age and immune status have a strong influence on pathogenic bacteria. Bacteria, fungi, viruses, and parasites can cause systemic inflammatory response syndrome, severe sepsis, and septic shock. Bacterial infection is the main cause of sepsis, with Gram-negative (G-) bacteria predominant before the 1980s, and the infection rate of Gram-positive ( $\mathrm{G}+$ ) bacteria gradually increased. The rates of $\mathrm{G}-$ and $\mathrm{G}+$ infections were comparable by the middle of the 1980s. To date, infections caused by G+ bacteria $(60 \%-70 \%)$ tend to exceed than those by G- bacteria $(28 \%-40 \%)[8,9]$. Neonatal pathogens are dominated by Staphylococcus $(\sim 60 \%)$, and coagulase-negative staphylococci is the most frequent pathogen (sequentially Staphylococcus epidermidis, Staphylococcus haemolyticus, and human Staphylococcus), followed by Klebsiella pneumoniae, Escherichia coli, and Acinetobacter baumannii [8-11]. For older children, coagulase-negative staphylococci ranks first, and except for the common neonatal pathogens, infections by Staphylococcus aureus, Enterococci, Salmonella, and Streptococcus show an increasing trend [12-14].

\section{Pathogen resistance}

At present, G+ and G- bacteria have poor sensitivity to ampicillin, tetracycline, and sulfamethoxazole, but G- bacteria are more sensitive to gentamicin and third-generation cephalosporins [9]. With the development of neonatal intensive care unit, mechanically assisted ventilation, long-term arteriovenous catheterization, and widespread use of broad-spectrum antibacterial agents, the infections caused by conditional pathogens (e.g. S. epidermidis and Klebsiella spp.) and drug-resistant bacteria have increased significantly, and the resistance to most commonly used antibacterial agents is severe. These infections exhibit multiple drug resistance [15]. We showed that coagulase-negative staphylococci are generally resistant to penicillin, ampicillin, oxacillin, and erythromycin but are more sensitive to vancomycin, linezolid, and teicoplanin $[14,16]$. The resistance rates of Klebsiella in Asia to ampicillin and cephalosporin antibiotics are $94 \%$ and $84 \%$, respectively, while the corresponding rates in Africa are $100 \%$ and 50\%. G- multidrug-resistant bacteria (resistant to ampicillin, chloramphenicol, and compound sulfamethoxazole) have accounted for $30 \%$ in Asia (interquartile ranges from $0.0 \%$ to $59.6 \%$ ) and $75 \%$ in Africa (interquartile ranges from $30.0 \%$ to $85.4 \%$ ) [17]. The distribution and drug resistance of pathogenic bacteria in the department may be quite distinct in specific hospitals.

\section{Determining and controlling the source of infection}

The definitive diagnosis of pathogens can effectively guide the targeted treatment and improve the cure rate. Prior to the anti-infective treatment, corresponding blood culture specimens and other possible microbial culture specimens (e.g. respiratory secretions, abscess fluid, urine, feces, cerebrospinal fluid, and other possible sources of infectious fluids) should be obtained. The sepsis infection originating from the respiratory system accounts for $52 \%$, while urogenital system, soft tissue, and abdominal infections account for $12 \%-14 \%$ [18]. The focus of infection cannot be rapidly and accurately determined in children because of the limited capabilities of expression and communication in this group. Under such conditions, imaging techniques are primarily used to check the focus of infection [19]. At the same time, the abdomen, armpit, 
cerebrospinal fluid, intestinal space, and other parts should be immediately checked. The focus of infection should be determined within $6 \mathrm{~h}$ of the patient's visit, and appropriate specimens should be obtained. Early control of the source of infection after fluid resuscitation in the early stages of sepsis can effectively improve patient survival. For identified abscesses, necrotic tissues should be promptly debrided and drained, and infected devices (such as catheter-related infections) should be promptly removed, avoiding the use of highrisk medical infection devices.

\section{Anti-infection treatment}

\subsection{Optimal timing}

Experimental data on pathogenic bacteria are difficult to obtain from children when they first enter the hospital because of the rapid onset and rapid progress of sepsis. An effective antibacterial drug for early treatment is also difficult to determine. Early, appropriate, and adequate initial empirical anti-infection treatment can prevent the progression of the disease and play an important role in the prognosis of the patient [20]. The doctor should administer empirical antimicrobial therapy as early as possible based on the judgment of the patient's condition combined with their clinical experience. Moreover, doctors should not wait for etiological results and miss the optimal timing for treatment. Once the patient has been diagnosed with sepsis or septic shock, intravenous antibiotics should be administered immediately within $1 \mathrm{~h}$ after the specimen is taken. Vascular access and blood collection are more difficult to perform in children than in adults. Thus, prior to the establishment of vascular access, the children should be administered intramuscular or oral antibiotics promptly if they are tolerant [6]. A retrospective study of 2,731 patients with septic shock showed that $79.9 \%$ of recovered and discharged patients were related to timely and effective use of antimicrobial agents within the first hour [21]. Early anti-infective therapy can significantly improve survival rate, while delayed anti-infective treatment is a risk factor that increases mortality [22]. Ferrer et al. [23] analyzed the empiric antibiotic use in 17,900 sepsis patients in 165 intensive care units in Europe, the United States, and South America from 2005 to 2010. The results showed that the mortality rate of patients administered with antibacterials within $1 \mathrm{~h}$ was $24.6 \%$, while the mortality rate increased with each $1 \mathrm{~h}$ delay of antibacterial drug administration, showing a steady growth trend. Administering antibiotics after $6 \mathrm{~h}$ resulted in the mortality rate of as high as $33.1 \%$.

\subsection{Selection of antibiotics}

Empirical medication need should be very cautiously dispensed for inconclusive pathogens because of the lack of specificity. Inaccurate judgments may cause unreasonable treatment and increase the risk of mortality of children. Doctors should consider microbiological epidemiological data and pathogen resistance, sensitivity, and other factors of the current, local, hospital, department, and use empirical antimicrobial agents early [24]. Moreover, whether the case is hospital-acquired or out-of-hospital infection should be determined, and the presence of a clear focus of infection should be known. The selected drug should be a wide spectrum of antimicrobial agent that covers possible pathogenic microorganisms (bacterial or fungal) and has good penetration in the likely infected tissue. The pharmacokinetics of the antimicrobials will change significantly during the course of treatment. Hence, close monitoring of blood levels is especially important [25], and antibiotics should be reasonably adjusted based on drug effects, pharmacokinetics, and liver and kidney function states.

After obtaining drug sensitivity test results and identifying the pathogens, appropriate narrow-spectrum antibacterial drug monotherapy should be selected to achieve targeted treatment in a step-by-step manner. In the 2012 edition of the International Guidelines for Treatment of Severe Sepsis and Septic Shock in Children, clindamycin and antitoxin therapy are recommended for patients with resistant hypotensive sepsis shock 
based on evidence-based grading criteria (level of evidence D). For patients with Clostridium difficile enteritis, intestinal antibiotic therapy is recommended with a high grade A evidence (if tolerated), and oral vancomycin can be chosen for severe conditions [6].

\subsection{Joint use of antimicrobial drugs}

In empirical anti-infective treatment, doctors often choose two or more antibacterial drugs to be used together to ensure that at least one antibacterial drug is sensitive to pathogens. However, whether the combined use of drug is more reasonable and effective remains controversial. A meta-analysis in 2010 showed that early administration of antibacterial drugs could increase the clinical response rate and survival rate in patients with sepsis [21]. An in vivo study on pigs used cefuroxime ( $\beta$-lactams) combined with bumycin (aminoglycoside) to treat severe E. coli infection. Compared with cefuroxime alone, the combined group increased the blood's lethality to bacteria and reduced the liver's bacteria. This result did not exclude the impact of difference in the ability of an individual's blood to kill bacteria [26]. However, in 2014, 69 randomized controlled trials were included in one study. The Cochrane systematic reviews reviewed and compared $\beta$-lactams + aminoglycosides and $\beta$-lactams in the treatment of sepsis among 7,863 subjects. The results showed that the mortality rate and clinical failure rate of single drug were lower than those of the combined group. The single-drug group had reduced renal toxicity. The researchers did not recommend combined use of $\beta$-lactam drugs and aminoglycosides for treatment, but such a combination was preferably recommended because of the low quality of evidence included [27]. Based on the above evidence and the complexity of the pathogens, the author believes that the current evidence is not sufficient to prove which therapy is better. Moreover, whether to use combined antibacterial drugs needs to be further confirmed by randomized controlled trials with a higher quality. In principle, single-agent therapy should be used as much as possible, and if necessary, drugs that can play a synergistic role should be selected in combined drugs.

\subsection{Course of antimicrobial medications}

Since empiric anti-infective treatment is conducted when pathogens are undetermined, overuse of antibiotics is often present. After $48-72 \mathrm{~h}$ of early sufficient anti-infective therapy, antibacterial drugs should be adjusted based on clinical effects, bacterial culture, and drug sensitivity test results. Narrow-spectrum antibacterial drugs should be dispensed as much as possible to prevent drug-resistant strains, reduce drug toxicity, and reduce medical costs. The current course of treatment for patients with severe sepsis needs in-depth study of antibiotics. Early stoppage of antibacterial drugs can reduce the occurrence of drug resistance, but the possibility of infection recurrence is increased. A long course of treatment will increase the risk of selective resistance [28]. Studies have confirmed that antibacterial agents should be stopped within 6-8 days for patients with severe infections. The course of medication for 7-10 days is reasonable and safe [29-31]. Proper extension of the course of treatment may be considered in cases of slow response to treatment, unclear infection focus, unsmooth drainage, and immune deficiency. Empiric combined use of antibiotics should not exceed 3-5 days as much as possible. Once drug susceptibility test results are obtained and the pathogen is identified, appropriate narrow-spectrum antibiotic monotherapy should be selected. The treatment regimen is closely observed for 3 days to reliably assess the efficacy of the regimen, during which the regimen should not be altered frequently.

The use of biomarkers to assist in decision-making during the administration of antibiotics has shown good advantages. This practice can guide rational drug use, reduce the use of antibiotics, and is relatively safe. Serum procalcitonin as a biomarker of infection can diagnose early sepsis, determine the severity of illness in children, and roughly identify pathogenic bacteria. Therefore, it can be used as an important indicator of bacterial infection and prognosis. Monitoring serum procalcitonin level can accurately and effectively guide the administration of antibiotics and shorten the course of medication [19,32,33]. Huang et al. [34] found that serum levels of procalcitonin in children with sepsis caused by G- bacterial infection were 
higher than those in G+-infection patients. This result provided a basis for the selection of antimicrobial agents. Oliveira et al. [30] also found that C-reactive protein and anti-procalcitonin could guide the antibacterial treatment of patients with severe sepsis. The median durations of antibiotics dispensed with guidance using the two markers were 6.0 days and 7.0 days, respectively $(P=0.06)$. The physical conditions, clinical symptoms, laboratory test results, and biomarkers of the children should be considered when determining when to stop administering antibiotics.

\section{Conclusion}

The antimicrobial drug abuse, increased drug-resistant bacteria, and lack of epidemiological data had resulted in the numerous challenges in the effective and reasonable anti-infective treatment of patients with sepsis, especially for children.

Based on currently available clinical evidence and expertise and experience of physicians, the patient's individual condition combined with $3+1$ evidence-based anti-infection treatment model of the local environment is an important concept for the successful rescue of patients with sepsis. Administration of antibacterial drugs and monitoring of drug resistance should be widely implemented in hospitals, regions, and countries. International and domestic hospitals should share epidemiological data. The awareness of clinicians on the rational administration of antibacterial drugs should be enhanced. Evidence-based localization guidelines are recommended for personnel training and education.

Acknowledgments: None.

Conflict of interest: The author states no conflicts of interest.

\section{References}

[1] Wiens M.O., Kumbakumba E., Kissoon N., Ansermino J.M., Ndamira A., Larson C.P., Pediatric sepsis in the developing world: challenges in defining sepsis and issues in post-discharge mortality, Clin. Epidemiol., 2012, 2012(4), 319-325.

[2] Institute for Health Metrics and Evaluation. GBD Compare. 2015 [2017-03-01]. http://vizhub.healthdata.org/gbd-compare/.

[3] Black R.E., Cousens S., Johnson H.L., Lawn J.E., Rudan I., Bassani D.G., et al., Global, regional, and national causes of child mortality in 2008: a systematic analysis, Lancet, 2010, 375(9730), 1969-1987.

[4] Filbin M.R., Arias S.A., Camargo C.A. Jr., Barche A., Pallin D.J., Sepsis visits and antibiotic utilization in U. S. emergency departments, Crit. Care Med., 2014, 42(3), 528-535.

[5] Bi L., Zhao De'an, Han Z., Current status of severe sepsis in children, Med. Philos, 2012, 33(6), 53-54.

[6] Dellinger R.P., Levy M.M., Rhodes A., Annane D., Gerlach H., Opal S.M., et al., Surviving sepsis campaign: international guidelines for management of severe sepsis and septic shock: 2012, Crit. Care Med., 2013, 41(2), 580-637.

[7] Yang H., The pathogenesis, diagnosis and treatment of sepsis in children, Int. J. Pediatr., 2014, 41(2), $138-141$.

[8] Zang W., Deng S., Analysis of pathogen distribution and drug susceptibility test results of 541 cases of neonatal blood culture, Mod. Med. Health, 2014, 30(1), 11-13; 16.

[9] Acquah S.E., Quaye L., Sagoe K., Ziem J.B., Bromberger P.I., Amponsem A.A., Susceptibility of bacterial etiological agents to commonly-used antimicrobial agents in children with sepsis at the Tamale Teaching Hospital, BMC Infect. Dis., 2013, 18(13), 89.

[10] Ramasamy S., Biswal N., Bethou A., Mathai B., Comparison of two empiric antibiotic regimen in late onset neonatal sepsis - a randomized controlled trial, J. Trop. Pediatr., 2014, 60(1), 83-86.

[11] Li Z., Xiao Z., Li Z., Zhong Q., Zhang Y., Xu F., 116 cases of neonatal early-onset or late-onset sepsis: a single center retrospective analysis on pathogenic bacteria species distribution and antimicrobial susceptibility, Int. J. Clin. Exp. Med., 2013, 6(8), 693-699.

[12] Wu X., Xiao S., Li B., Ni L., Huang H., Analysis of bacterial pathogens and drug resistance in children with septicemia in Kunming Children's Hospital from 2009 to 2011, Chin. J. Clin. Doctors Electron. Ed., 2013, 7(13), 6090-6092.

[13] Hou H., Gao L., Wang X., Chen W., Analysis of distribution and drug resistance of pathogens in children's blood culture in Nanjing from 2009 to 2011, Lab. Med., 2013, 28(11), 1030-1033. 
[14] Cai X., Lv X., Qing Z., Analysis of distribution and drug resistance of pathogens in children's blood culture, Int. J. Lab.Med., 2012, 33(13), 1613-1614; 1617.

[15] Ganatra H.A., Zaidi A.K., Neonatal infections in the developing world, Semin. Perinatol., 2010, 34(6), 416-425.

[16] Zhao J., Xiong T., Tang J., Mu D., Clinical features of early-onset and late-onset neonatal sepsis, J. Appl.Pediatr., 2012, 27(10), 736-738.

[17] Le Doare K., Bielicki J., Heath P.T., Sharland M., Systematic review of antibiotic resistance rates among Gram-negative bacteria in children with sepsis in resource-limited countries, J Pediatric Infect. Dis. Soc, 2015, 4(1), 11-20.

[18] Nygård S.T., Langeland N., Flaatten H.K., Fanebust R., Haugen O., Skrede S., Aetiology, antimicrobial therapy and outcome of patients with community acquired severe sepsis: a prospective study in a Norwegian university hospital, BMC Infect. Dis., 2014, 4(14), 121.

[19] Agarwal R., Schwartz D.N., Procalcitonin to guide duration of antimicrobial therapy in intensive care units: a systematic review, Clin. Infect. Dis., 2011, 53(4), 379-387.

[20] Paul M., Shani V., Muchtar E., Kariv G., Robenshtok E., Leibovici L., Systematic review and meta-analysis of the efficacy of appropriate empiric antibiotic therapy for sepsis, Antimicrob. Agents Chemother., 2010, 54(11), 4851-4863.

[21] Kumar A., Safdar N., Kethireddy S., Chateau D., A survival benefit of combination antibiotic therapy for serious infections associated with sepsis and septic shock is contingent only on the risk of death: a meta-analytic/meta-regression study, Crit. Care Med., 2010, 38(8), 1651-1664.

[22] Jalili M., Barzegari H., Pourtabatabaei N., Honarmand A.R., Boreiri M., Mehrvarz A., et al., Effect of door-to-antibiotic time on mortality of patients with sepsis in emergency department: a prospective cohort study, Acta Med. Iran., 2013, 51(7), 454-460.

[23] Ferrer R., Martin-Loeches I., Phillips G., Osborn T.M., Townsend S., Dellinger R.P., et al., Empiric antibiotic treatment reduces mortality in severe sepsis and septic shock from the first hour: results from a guideline-based performance improvement program, Crit. Care Med., 2014, 42(8), 1749-1755.

[24] Randolph A.G., Mcculloh R.J., Pediatric sepsis: important considerations for diagnosing and managing severe infections in infants, children, and adolescents, Virulence, 2014, 5(1), 179-189.

[25] Duszyńska W., Strategies of empiric antibiotic therapy in severe sepsis, Anaesthesiol. Intensive Ther., 2012, 44(2), 96-103.

[26] Skorup P., Maudsdotter L., Lipcsey M., Castegren M., Larsson A., Jonsson A.B., et al., Beneficial antimicrobial effect of the addition of an aminoglycoside to a $\beta$-lactam antibiotic in an $E$. coli porcine intensive care severe sepsis model, PLoS ONE, 2014, 9(2), e90441.

[27] Paul M., Lador A., Grozinsky-Glasberg S., Leibovici L., Beta lactam antibiotic monotherapy versus beta lactamaminoglycoside antibiotic combination therapy for sepsis, Cochrane Database Syst. Rev., 2014, 7(1), CD003344.

[28] Horisberger T., Harbarth S., Nadal D., Baenziger O., Fischer J.E., G-CSF and IL-8 for early diagnosis of sepsis in neonates and critically ill children-safety and cost effectiveness of a new laboratory prediction model: study protocol of a randomized controlled trial [ISRCTN91123847], Crit. Care, 2004, 8(6), R443-R450.

[29] Dimopoulos G., Poulakou G., Pneumatikos I.A., Armaganidis A., Kollef M.H., Matthaiou D.K., Short- vs long-duration antibiotic regimens for ventilator-associated pneumonia: a systematic review and meta-analysis, Chest, 2013, 144(6), 1759-1767.

[30] Oliveira C.F., Botoni F.A., Oliveira C.R., Silva C.B., Pereira H.A., Serufo J.C., et al., Procalcitonin versus C-reactive protein for guiding antibiotic therapy in sepsis: a randomized trial, Crit. Care Med., 2013, 41(10), 2336-2343.

[31] Dellinger R.P., Levy M.M., Carlet J.M., Bion J., Parker M.M., Jaeschke R., et al., Surviving sepsis campaign: international guidelines for management of severe sepsis and septic shock: 2008, Intensive Care Med., 2008, 34(1), 17-60.

[32] Bouadma L., Luyt C.E., Tubach F., Cracco C., Alvarez A., Schwebel C., et al., Use of procalcitonin to reduce patients' exposure to antibiotics in intensive care units (PRORATA trial): a multicentre randomised controlled trial, Lancet, 2010, 375(9713), 463-474.

[33] Schuetz P., Chiappa V., Briel M., Greenwald J.L., Procalcitonin algorithms for antibiotic therapy decisions: a systematic review of randomized controlled trials and recommendations for clinical algorithms, Arch. Intern. Med., 2011, 171(15), 1322-1331.

[34] Huang C., Mo L., Li X., Jiang Y., Zhou Z., Serum procalcitonin levels in children with sepsis due to different bacterial infections, Int. J. Lab. Med., 2010, 31(10), 1110-1111. 